\title{
Endocarditis as the first presentation of AIDS in infancy
}

\author{
Catharina A M van Doorn, Robert Yates, Victor T Tsang
}

\begin{abstract}
A two month old Ugandan boy underwent surgery for an obstructive right ventricular vegetation associated with disseminated Staphylococcus aureus infection. Both the child and his mother subsequently tested positive for HIV infection. Very little is know about the incidence of endocarditis in paediatric patients with AIDS. To our knowledge this is the first case reported of disseminated $S$ aureus infection associated with endocarditis and an obstructing vegetation in an HIV positive infant with a structurally normal heart. The initial signs and symptoms for endocarditis were atypical, a reflection of the overwhelming infection in an immunocompromised patient. Severe infections may have an atypical presentation in immunosuppressed patients. AIDS needs to be considered in these patients, especially if they come from populations with endemic HIV infection.

(Arch Dis Child 1998;79:179-180)
\end{abstract}

Keywords: AIDS; HIV; endocarditis

A 2 month old boy presented to his local hospital with a two day history of sticky eyes, tachypnoea, and a high pitched cry. He was the first child of Ugandan parents. He had been born in England by caesarian section because of an unfavourable lie, but there had been no prenatal or postnatal problems. On physical examin-

Accepted 20 January 1998

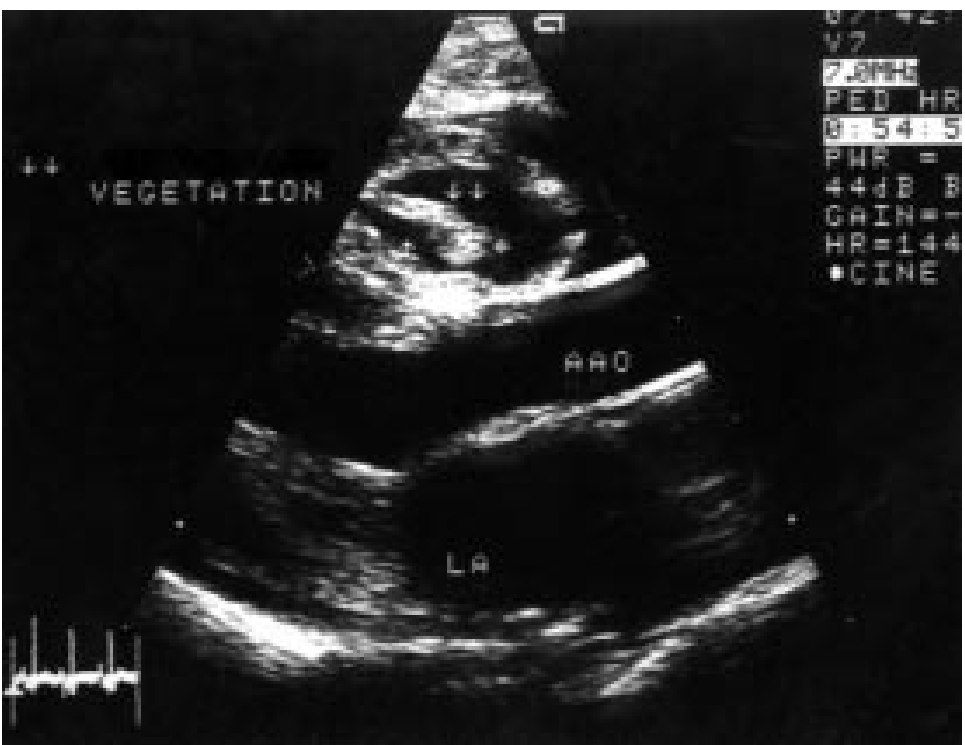

Figure 1 Echocardiogram (parasternal long axis view) showing a long pedunculated vegetation (arrows) attached to the free wall of the right ventricle. Note pericardial effusion anterior to the right ventricle. AAO, ascending aorta; $L A$, left atrium. ation he appeared well and was breast feeding without problems. His temperature was $38^{\circ} \mathrm{C}$, both his eyes were sticky, and he had a small easily reducible umbilical hernia but otherwise examination was unremarkable. He was admitted, initially for observation, but became acutely unwell overnight with tachypnoea and poor perfusion. On repeat examination there were scattered lung crackles, a grade $2 / 6$ systolic murmur over the left sternal edge, and a $4 \mathrm{~cm}$ hepatomegaly. Blood pressure was $85 /$ $50 \mathrm{~mm} \mathrm{Hg}$ and femoral pulses were easily palpable. Haemoglobin $(63 \mathrm{~g} / \mathrm{l})$ and platelet count $\left(35 \times 10^{9} / 1\right)$ were low, white cell count was $7.8 \times 10^{9} / 1$ with toxic granulation of neutrophils $\left(4.83 \times 10^{9} / 1\right)$ and lymphopenia $\left(0.39 \times 10^{9} / 1\right)$. He had hyponatraemia $(116 \mathrm{mmol} / \mathrm{l})$ with normal serum potassium and renal function. His clotting times were prolonged with an activated partial thromboplastin time of 120 seconds (normal 33 seconds) and thrombin time of 60 seconds (normal 10 seconds). In addition, fibrinogen degradation products were severely raised (1050 ng/l; normal 0-250 ng/1). Fibrinogen concentrations and partial thrombin time were normal. Chest radiography showed cardiomegaly and left pleural effusion. Electrocardiography showed right bundle branch pattern and left ventricular hypertrophy. Echocardiography suggested a mass in the right ventricle. Differential diagnosis included cardiac tumour, thrombus with hypercoagulability syndrome, or infective endocarditis and he was transferred to our institution for further management.

Detailed echocardiography on admission demonstrated a structurally normal heart apart from a patent foramen ovale. There was a pedunculated mass attached to the free wall of the right ventricle (fig 1), which prolapsed through the pulmonary valve and intermittently obstructed the right ventricular outflow tract (fig 2). Because of further deterioration in his condition the child was referred for urgent surgery. At operation there was a purulent pericardial effusion, multiple infected foci in the lung, as well as an empyema in the left pleural cavity, which was drained. On cardiopulmonary bypass a large, mobile vegetation was excised from the right ventricle and the patent foramen ovale was closed. In the immediate postoperative period haemodynamic support with inotropes and vasoconstrictors was required for shock.

Cultures from the cardiac vegetation, pleural fluid, and peripheral blood cultures grew Staphylococcus aureus and the child was treated with flucloxacillin, fucidic acid, and gentamicin according to sensitivity. Blood cultures became 


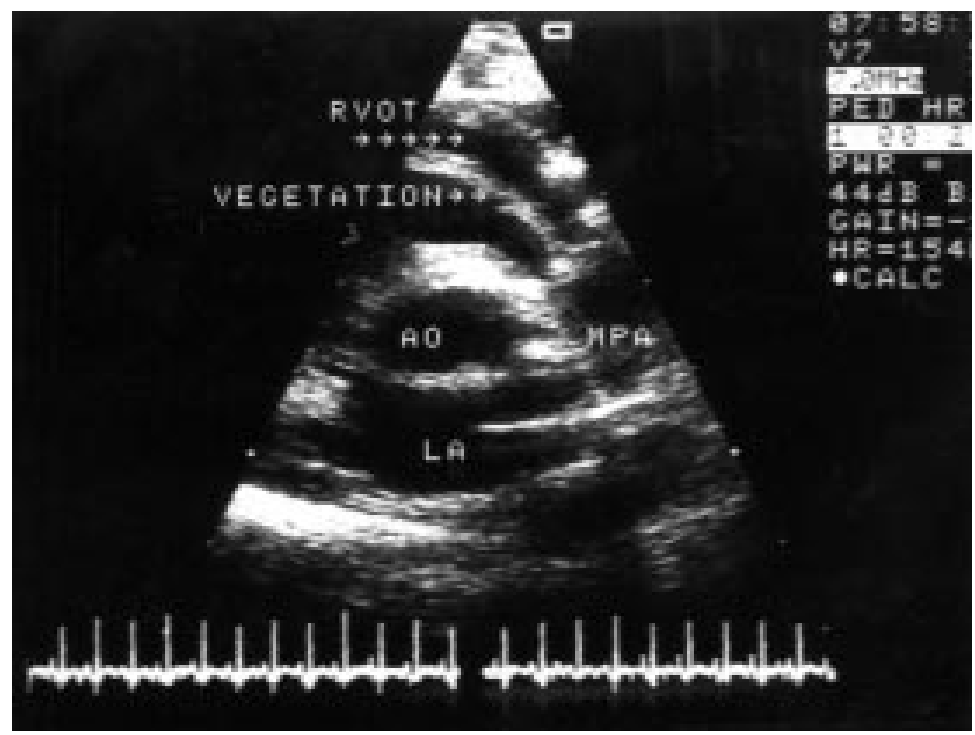

Figure 2 Echocardiogram (parasternal short axis view) showing vegetation (arrows) prolapsing through the pulmonary valve into the main pulmonary artery. LA, left atrium; $M P A$, main pulmonary artery; AO, ascending aorta; RVOT, right ventricular outflow tract.

negative on the seventh postoperative day. Because of pneumonia and recurrent pulmonary oedema, the child remained ventilator dependent until the 24th postoperative day. During this period he also required large doses of diuretics and developed hyponatraemia and hypokalaemia associated with cardiac arrhythmias but these resolved after correction of the electrolyte balance. He then had an episode of Escherichia coli sepis, for which ciprofloxacin was added to the antibiotic regimen, and a Candida albicans urinary tract infection.

In view of the severe, disseminated staphylococcus infection, investigations for primary and acquired immune deficiency were performed. A nitro blue tetrazolium test was normal, immunoglobulin $\mathrm{E}$ was raised $(13.8 \mathrm{kU} / \mathrm{l})$, and a lymphocyte subset panel showed decreased $\mathrm{B}$ cells. Both mother and child tested positive for HIV 1 and HIV 2 antibody. A polymerase chain reaction test on the child's serum was positive for HIV 1 genome; p24 antigen testing was also positive. Bronchoalveolar lavage revealed $S$ aureus but showed no evidence of acid fast bodies, legionella, mycoplasma, parainfluenza A and B, measles, or Pneumocystis carinii. Viral serology as well as bronchoalveolar lavage were negative for adenovirus, influenza $\mathrm{A}$ and $\mathrm{B}$, respiratory syncytial virus, and Mycoplasma pneumoniae. Culture of cerebrospinal fluid was negative. An ultrasound scan of the abdomen and head revealed no focus of infection.

The child was transferred back to the referring hospital on the 32nd postoperative day. His cardiovascular status was stable, oxygen saturations were $94 \%$ on 2 litres/min of nasal oxygen, and he was absorbing nasogastric feeds. On review in the outpatient clinic four weeks later he was well but remained tachypnoeic. His current antiretroviral treatment includes nelfinavir $150 \mathrm{mg}$ three times a day, stavudine $6.5 \mathrm{mg}$ twice a day, didanosine $35 \mathrm{mg}$ twice a day, and cotrimoxazole $240 \mathrm{mg}$ three times a week. Further testing of the child's serum continues to demonstrate a high viral load.

\section{Discussion}

Very little is known about the incidence of endocarditis in paediatric patients with AIDS. To our knowledge this is the first case reported of disseminated $S$ aureus infection associated with endocarditis and an obstructing vegetation in an HIV positive infant with a structurally normal heart. However, endocarditis is well recognised in HIV positive patients who are intravenous drug users and typically occurs in the right sided circulation. ${ }^{1}$ In these patients $S$ aureus is the most frequently identified organism. ${ }^{2}$

Our patient most likely acquired AIDS through vertical transmission and remained well during the first two months of life. The initial signs and symptoms for endocarditis were atypical, a reflection of the overwhelming infection in an immunocompromised patient. The child then developed acute cardiac failure because of the obstructive nature of the large vegetation, and infective endocarditis was first considered when a ventricular mass was identified using echocardiography. The nature of the infection, however, especially in a patient from a population with a high prevalence of AIDS, suggested that acquired immunodeficiency might play a role. The primary focus for the staphylococcus infection in this case could not be identified.

Urgent surgical resection of the mass was indicated for relief of life threatening cardiac obstruction but was also useful for diagnostic reasons. In adult patients with known endocarditis and non-obstructing vegetations, antibiotic treatment alone has been successful in controlling the infection. However, embolisation of the vegetation and subsequent development of pulmonary hypertension has been observed. ${ }^{3}$

Severe infections may have an atypical presentation in immunosuppressed patients. AIDS needs to be considered in these patients, especially if they come from populations with endemic HIV infection.

1 Currie PF, Sutherland GR, Jacobs AJ, Brettle RP, Boon NA. A review of endocarditis in acquired immunodeficiency Eyndrome and human immunodeficiency virus infection.

2 Nahass RG, Weinstein MP, Bartels J, Gocke DJ. Infective endocarditis in intravenous drug users: a comparison of endocarditis in intravenous drug users: a comparison of
human immuno deficiency virus type I-negative and human immuno deficiency virus type I-n

3 De Castro S, Migliau G, Silvestri A, et al. Heart involvement in AIDS: a prospective study during various stages of the disease. Eur Heart f 1992;13:1452-9. 